\title{
Matrix Mappings on the Domains of Invertible Matrices
}

\author{
Muhammed Altun \\ Faculty of Arts and Sciences, Melikşah University, 38280 Kayseri, Turkey \\ Correspondence should be addressed to Muhammed Altun; muhammedaltun@gmail.com
}

Received 31 May 2013; Accepted 5 September 2013

Academic Editor: Kasso A. Okoudjou

Copyright (C) 2013 Muhammed Altun. This is an open access article distributed under the Creative Commons Attribution License, which permits unrestricted use, distribution, and reproduction in any medium, provided the original work is properly cited.

We focus on sequence spaces which are matrix domains of Banach sequence spaces. We show that the characterization of a random matrix operator $T=\left(t_{n k}\right) \in\left(E_{A}, F_{B}\right)$, where $E_{A}$ and $F_{B}$ are matrix domains with invertible matrices $A$ and $B$, can be reduced to the characterization of the operator $S=B \circ T \circ A^{-1} \in(E, F)$. As an application, the necessary and sufficient conditions for the matrix operators between invertible matrix domains of the classical sequence spaces and norms of these operators are given.

\section{Introduction and Preliminaries}

Let $\omega$ denote the set of all complex sequences. Any subspace of $\omega$ is called as a sequence space. We will write $\ell_{\infty}, c$, and $c_{0}$ for the spaces of all bounded, convergent, and null sequences, respectively. By $\ell_{p}$, we denote the space of all $p$ absolutely summable sequences, where $1 \leq p<\infty$.

Let $X$ and $Y$ be Banach spaces. Then, $(X, Y)$ is the set of all operators $L: X \rightarrow Y$ and $\mathscr{B}(X, Y)$ is the set of all continuous linear operators $L: X \rightarrow Y . \mathscr{B}(X, Y)$ is a Banach space with the operator norm defined by $\|L\|=\sup \{\|L(x)\| /\|x\|: 0 \neq x \in$ $X\}(L \in \mathscr{B}(X, Y))$.

Let $E$ and $F$ be two sequence spaces and $A=\left(a_{n k}\right)$ an infinite matrix of real or complex numbers $a_{n k}$, where $n$, $k \in \mathbb{N}=\{1,2,3, \ldots\}$. Then, we say that $A$ defines a matrix mapping from $E$ into $F$, and we denote it by writing $A$ : $E \rightarrow F$, if for every sequence $x=\left(x_{k}\right) \in E$ the sequence $A x=\left\{(A x)_{n}\right\}$, the $A$-transform of $x$, is in $F$, where

$$
(A x)_{n}=\sum_{k} a_{n k} x_{k} \quad(n \in \mathbb{N}) .
$$

Thus, $A \in(E, F)$ if and only if the series on the right side of (1) converges for each $n \in \mathbb{N}$ and every $x \in E$, and we have $A x=\left\{(A x)_{n}\right\}_{n \in \mathbb{N}} \in F$ for all $x \in E$.

Let $A=\left(a_{n k}\right)$ and $B=\left(b_{n k}\right)$. Suppose the sums

$$
c_{n k}=\sum_{j} a_{n j} b_{j k}
$$

exist for all $n, k \in \mathbb{N}$. Then, the product of $A$ and $B$ is defined by $A B=\left(c_{n k}\right)$.

If $E$ is a subset of $\omega$, then $E_{A}=\{x \in \omega: A x \in E\}$ is the matrix domain of $A$ in $E$. We will say that a matrix $A$ is invertible over a sequence space $E$ if the operator $A: E_{A} \rightarrow$ $E$ is bijective; that is, there exists an operator $A^{-1}$ such that $A^{-1}(A x)=x$ for all $x \in E_{A}$ and $A\left(A^{-1} y\right)=y$ for all $y \in E$. We will say $A$ is invertible if $A$ is invertible over $\omega$.

A BK space is a Banach sequence space with continuous coordinates. The sequence spaces $c_{0}, c, \ell_{p}$, and $\ell_{\infty}$ are the wellknown examples of BK spaces.

Several authors studied matrix mappings on sequence spaces that are matrix domains of the difference operator or of the matrices of some classical methods of summability in spaces such as $\ell_{p}, c_{0}, c$, or $\ell_{\infty}$. For instance, some matrix domains of the difference operator were studied in $[1,2]$, of the Cesàro matrices in $[3,4]$, of the Euler matrices in $[5-$ 7], and of the Nörlund matrices in [8]. A general approach was done in [9] reducing the characterizations of the classes $\left(X_{T}, Y\right)$ for arbitrary $\mathrm{FK}$ spaces $X$ with $\mathrm{AK}$ and $Y \subset \omega$ to those of the classes $(X, Y)$ and $(X, c)$, where $T$ is a triangle. Compact operators on matrix domains of triangles were examined in [10]. The gliding hump properties of matrix domains were examined in [11].

In this work, our aim is to give some general results for matrix mappings between sequence spaces, which are matrix domains of invertible matrices of sequence spaces. Also, we give some applications of the results. 
Theorem 1 (see [12, Theorem 4.2.8, page 57]). Matrix operators between $B K$ spaces are continuous.

An infinite matrix $T=\left(t_{n k}\right)$ is said to be a triangle if $t_{n k}=$ 0 for $n<k$ and $t_{n n} \neq 0$ for $n \in \mathbb{N}$. The following is a wellknown result about triangles.

Theorem 2 (see $[12,1.4 .8$, page 9]). Every triangle $T$ has an inverse $T^{-1}$ which also is a triangle, and $x=T\left(T^{-1} x\right)=$ $T^{-1}(T x)$ for all $x \in \omega$.

Let $A_{1}, A_{2}$ be two matrices and $\mu$ a sequence space. If $A_{1}\left(A_{2} x\right)=\left(A_{1} A_{2}\right) x$ holds for all $x \in \mu$, then we will say $A_{1}$ and $A_{2}$ are associative over the space $\mu$. If $A_{1}$ and $A_{2}$ are associative over the space $\omega$, we will shortly say $A_{1}$ and $A_{2}$ are associative. For row finite matrices, we do not generally have an inverse. But we have associativity; that is, for any two row finite matrices $R_{1}$ and $R_{2}$ we have

$$
R_{1}\left(R_{2} x\right)=\left(R_{1} R_{2}\right) x \quad \forall x \in \omega .
$$

Moreover, we have the following result.

Theorem 3 (see [12, Theorem 1.4.4, page 8]). Let $A_{1}, A_{2}$ be two matrices.

(i) If rows of $A_{1}$ are in $\ell_{1}$ and $A_{2} \in\left(\ell_{\infty}, \ell_{\infty}\right)$, then $A_{1}$ and $A_{2}$ are associative over $\ell_{\infty}$.

(ii) If $A_{1}$ is row finite, then $A_{1}$ and $A_{2}$ are associative over $\omega_{A_{2}}$.

Corollary 4. The set of matrices $\left(\ell_{\infty}, \ell_{\infty}\right)$ are associative over $\ell_{\infty}$

Remark 5. The associativity property does not hold in general (see, e.g., [12, Example 1.4.6]).

Theorem 6. Let $A$ be an invertible matrix over a normed sequence space $E$ with norm $\|\cdot\|_{E}$. Then, $E_{A}$ is a normed sequence space with norm $\|z\|_{E_{A}}=\|A z\|_{E}$.

Proof. Let $\alpha \in \mathbb{C}$ and $z \in E_{A}$. Then,

$$
\begin{aligned}
\|\alpha z\|_{E_{A}} & =\|A(\alpha z)\|_{E}=\|\alpha A z\|_{E} \\
& =|\alpha|\|A z\|_{E}=|\alpha|\|z\|_{E_{A}} .
\end{aligned}
$$

Secondly, for $y, z \in E_{A}$ we have

$$
\begin{aligned}
\|y+z\|_{E_{A}} & =\|A(y+z)\|_{E} \\
& =\|A y+A z\|_{E} \leq\|A y\|_{E}+\|A z\|_{E} \\
& =\|y\|_{E_{A}}+\|z\|_{E_{A}},
\end{aligned}
$$

so the triangle inequality holds.

Now, suppose that $\|z\|_{E_{A}}=0$. Then, $\|A z\|_{E}=0$ and since $\|\cdot\|_{E}$ is a norm we have $A z=\theta$. Since $A$ is invertible, we have $z=\theta$.

Theorem 7. Let $E$ be a Banach sequence space and A a matrix. Then, the operator $A: E_{A} \rightarrow E$ is linear and continuous.
Proof. Let $A_{n}$ be the operator that corresponds to the $n$th row of the matrix operator $A$; that is, $A_{n} x=\sum_{k=1}^{\infty} a_{n k} x_{k}$ for all $x=\left(x_{k}\right) \in E_{A}$. Let $x=\left(x_{k}\right) \in E_{A}$ and $y=\left(y_{k}\right) \in E_{A}$. Clearly, we have $A_{n} c x=c A_{n} x$ for any $c \in \mathbb{C}$. Let $x^{\prime}=$ $A_{n} x=\sum_{k=1}^{\infty} a_{n k} x_{k}$ and $y^{\prime}=A_{n} y=\sum_{k=1}^{\infty} a_{n k} y_{k}$. We have $A_{n}(x+y)=\sum_{k=1}^{\infty} a_{n k}\left(x_{k}+y_{k}\right)=\lim _{m} \sum_{k=1}^{m} a_{n k}\left(x_{k}+y_{k}\right)=$ $\lim _{m} \sum_{k=1}^{m} a_{n k} x_{k}+\lim _{m} \sum_{k=1}^{m} a_{n k} y_{k}=x^{\prime}+y^{\prime}=A_{n} x+A_{n} y$. That means the operator $A_{n}$ is linear for arbitrary $n$, which implies the linearity of $A$.

$A$ is continuous since

$$
\begin{aligned}
\|A\| & =\sup \left\{\frac{\|A x\|_{E}}{\|x\|_{E_{A}}}: 0 \neq x \in E_{A}\right\} \\
& =\sup \left\{\frac{\|A x\|_{E}}{\|A x\|_{E}}: 0 \neq x \in E_{A}\right\}=1 .
\end{aligned}
$$

\section{Main Results}

Theorem 8. If $(E,\|\cdot\|)$ is a Banach sequence space and $A$ is an invertible matrix over $E$, then $\left(E_{A},\|\cdot\|_{A}\right)$ is a Banach space.

Proof. Let $(E,\|\cdot\|)$ be a Banach sequence space. Then, $\left(E_{A},\|\cdot\|_{A}\right)$ is a normed space by the previous theorem. Now, let $\left(x_{n}\right)$ be a Cauchy sequence in $E_{A}$. Let $y_{n}=A x_{n}$. Then $\left(y_{n}\right)$ is a sequence in $E$ and is Cauchy in $E$ since

$$
\begin{aligned}
\left\|x_{n}-x_{m}\right\|_{E_{A}} & =\left\|A\left(x_{n}-x_{m}\right)\right\|_{E} \\
& =\left\|A x_{n}-A x_{m}\right\|_{E}=\left\|y_{n}-y_{m}\right\|_{E} .
\end{aligned}
$$

Let $y \in E$ such that $\lim y_{n}=y$ in $E$. Let $x=A^{-1} y$. Then,

$$
\begin{aligned}
\lim _{n \rightarrow \infty}\left\|x_{n}-x\right\|_{E_{A}} & =\lim _{n \rightarrow \infty}\left\|A\left(x_{n}-x\right)\right\|_{E} \\
& =\lim _{n \rightarrow \infty}\left\|y_{n}-y\right\|_{E}=0 .
\end{aligned}
$$

Theorem 9. Let $A$ and $B$ be invertible matrices over the $B K$ spaces $E$ and $F$, respectively. Suppose that $T \in\left(E_{A}, F_{B}\right)$. If $B$ 。 $T \circ A^{-1}=B T A^{-1}$ over $E$, then the operator $T \in\left(E_{A}, F_{B}\right)$ is continuous.

Proof. Let $T \in\left(E_{A}, F_{B}\right)$ and let $\|\cdot\|$ be the norm of $E$. Since $A$ is invertible over $E, A: E_{A} \rightarrow E$ is bijective. Also, $A$ is continuous by Theorem 7 .

By the bounded inverse theorem $A^{-1}$ is continuous. Similarly the inverse $B^{-1}$ of $B: F_{B} \rightarrow F$ is continuous.

Now, we have that the matrix operator $R=B \circ T \circ A^{-1} \epsilon$ $(E, F)$ and is continuous by Theorem 1 . Since $A$ and $B^{-1}$ are continuous, the operator $T=B^{-1} \circ R \circ A$ is continuous.

Theorem 10. Let $A$ and $B$ be invertible matrices over the sequence spaces $E$ and $F$, respectively. Then, for an operator $T$, $T \in\left(E_{A}, F_{B}\right)$ if and only if $B \circ T \circ A^{-1} \in(E, F)$. 
Proof. Suppose that $T \in\left(E_{A}, F_{B}\right)$, and let $z=\left(z_{k}\right) \in E$. Then, clearly $A^{-1} z \in E_{A}$. Hence, we have $\left(T \circ A^{-1}\right) z \in F_{B}$ and so $\left(B \circ T \circ A^{-1}\right) z \in F$.

For the inverse implication, suppose that $B \circ T \circ A^{-1} \in$ $(E, F)$, and let $z \in E_{A}$. Then, $A z \in E$ and so $\left(B \circ T \circ A^{-1} \circ A\right) z=$ $(B \circ T) z \in F$. Since $B$ is invertible, we have $T z \in F_{B}$.

Corollary 11. Let $E$ and $F$ be sequence spaces and $A$ and $B$ triangles. Then, for an operator $T, T \in\left(E_{A}, F_{B}\right)$ if and only if $B \circ T \circ A^{-1} \in(E, F)$.

Theorem 12. Let $E$ and $F$ be two normed sequence spaces and $A$ and $B$ invertible matrices over $E$ and $F$, respectively. Then, for an operator $T, T \in \mathscr{B}\left(E_{A}, F_{B}\right)$ if and only if $B \circ T \circ A^{-1} \in$ $\mathscr{B}(E, F)$. In this case, one has

$$
\|T\|_{\left(E_{A}, F_{B}\right)}=\left\|B \circ T \circ A^{-1}\right\|_{(E, F)} .
$$

Proof. It is enough to show the following equality:

$$
\begin{aligned}
\|T\|_{\left(E_{A}, F_{B}\right)} & =\sup _{\theta \neq z \in E_{A}} \frac{\|T z\|_{F_{B}}}{\|z\|_{E_{A}}}=\sup _{\theta \neq A z \in E} \frac{\|(B \circ T) z\|_{F}}{\|A z\|_{E}} \\
& =\sup _{\theta \neq A z \in E} \frac{\left\|\left(B \circ T \circ A^{-1} \circ A\right) z\right\|_{F}}{\|A z\|_{E}} \\
& =\left\|\left(B \circ T \circ A^{-1}\right)\right\|_{E, F} .
\end{aligned}
$$

\section{Examples and Applications}

Theorem 10, Corollary 11, and Theorem 12 have many applications, especially in the subject of characterization of matrices which act as operators between certain sequence spaces. We just give a taste by the following examples and theorems.

Example 13. Let $\lambda=\left(\lambda_{k}\right)$ be a sequence of nonzero scalars in $\mathbb{C}$. For any $z=\left(z_{k}\right) \in \omega$, let $\lambda z=\left(\lambda_{k} z_{k}\right)$. Then, for a sequence space $E$, the multiplier sequence space $E(\lambda)$, associated with the multiplier sequence $\lambda$, is defined as

$$
E(\lambda)=\{z \in \omega: \lambda z \in E\}
$$

Let

$$
\Lambda=\left[\begin{array}{ccccc}
\lambda_{1} & 0 & 0 & 0 & \cdots \\
0 & \lambda_{2} & 0 & 0 & \cdots \\
0 & 0 & \lambda_{3} & 0 & \cdots \\
0 & 0 & 0 & \lambda_{4} & \cdots \\
\vdots & \vdots & \vdots & \vdots & \ddots
\end{array}\right]
$$

Then, the matrix $\Lambda$ is invertible with

$$
\Lambda^{-1}=\left[\begin{array}{ccccc}
\lambda_{1}^{-1} & 0 & 0 & 0 & \cdots \\
0 & \lambda_{2}^{-1} & 0 & 0 & \cdots \\
0 & 0 & \lambda_{3}^{-1} & 0 & \cdots \\
0 & 0 & 0 & \lambda_{4}^{-1} & \cdots \\
\vdots & \vdots & \vdots & \vdots & \ddots
\end{array}\right]
$$

and $E(\lambda)=E_{\Lambda}$. So, all the Theorems in Section 2 are applicable for multiplier sequence spaces (see [13] for detailed applications and examples).

Example 14. Let $T=\left(t_{n k}\right)$ be a row-finite matrix and $B$ the operator with matrix representation

$$
B=\left[\begin{array}{ccccc}
1 & 0 & 0 & 0 & \cdots \\
1 & 1 & 0 & 0 & \cdots \\
1 & 1 & 1 & 0 & \cdots \\
1 & 1 & 1 & 1 & \cdots \\
\vdots & \vdots & \vdots & \vdots & \ddots
\end{array}\right] .
$$

$B$ is invertible and the inverse operator has the matrix representation

$$
B^{-1}=\left[\begin{array}{ccccc}
1 & 0 & 0 & 0 & \cdots \\
-1 & 1 & 0 & 0 & \cdots \\
0 & -1 & 1 & 0 & \cdots \\
0 & 0 & -1 & 1 & \cdots \\
\vdots & \vdots & \vdots & \vdots & \ddots
\end{array}\right] .
$$

$B$ and $B^{-1}$ are triangles and so they are one to one. $B^{-1}$ is the operator $B(r, s)$ of [14] with $r=1$ and $s=-1$. Since both $T$ and $B^{-1}$ are row finite, we have $T \circ B^{-1}=T B^{-1}$. Now, using Corollary 11 we have that $T$ is in $\left(c_{B}, c\right)$ if and only if $T B^{-1}$ is in $(c, c)$ :

$$
\left[T B^{-1}\right]_{n k}=t_{n k}-t_{n(k+1)},
$$

so by the Kojima-Schur Theorem (see, e.g., Theorem 2.7 of [15]) we have that $T \in\left(c_{B}, c\right)$ if and only if the following three conditions hold:

(i) $\lim _{n} t_{n k}-t_{n(k+1)}$ exists for each $k$;

(ii) $\lim _{n} \sum_{k} t_{n k}-t_{n(k+1)}$ exists;

(iii) $\sup _{n} \sum_{k}\left|t_{n k}-t_{n(k+1)}\right|<\infty$.

Now, let us examine $\left(c, c_{B}\right) . T$ is in $\left(c, c_{B}\right)$ if and only if $B T$ is in $(c, c)$. We have

$$
[B T]_{n k}=s_{n k},
$$

where $s_{n k}$ is defined as the sum of the first $n$ terms of the $k$ th column of the matrix $T$; that is,

$$
s_{n k}=t_{1 k}+t_{2 k}+\cdots+t_{n k} .
$$

Now, by using the Kojima-Schur Theorem, we have, $T \in$ $\left(c, c_{B}\right)$ if and only if the following three conditions hold:

(i) $\lim _{n} s_{n k}$ exists for each $k$;

(ii) $\lim _{n} \sum_{k} s_{n k}$ exists;

(iii) $\sup _{n} \sum_{k}\left|s_{n k}\right|<\infty$.

Now, let us examine $\left(c_{B}, c_{B}\right) . T$ is in $\left(c_{B}, c_{B}\right)$ if and only if $B T B^{-1}$ is in $(c, c)$. We have

$$
\left[B T B^{-1}\right]_{n k}=s_{n k}-s_{n(k+1)} .
$$


Now, by using the Kojima-Schur Theorem we have that $T \in$ $\left(c_{B}, c_{B}\right)$ if and only if the following three conditions hold:

(i) $\lim _{n} s_{n k}-s_{n(k+1)}$ exists for each $k$;

(ii) $\lim _{n} \sum_{k} s_{n k}-s_{n(k+1)}$ exists;

(iii) $\sup _{n} \sum_{k}\left|s_{n k}-s_{n(k+1)}\right|<\infty$.

Theorem 15. An infinite matrix $A=\left(a_{n k}\right) \in\left(\ell_{\infty}, b v\right)$ if and only if

$$
\sup _{N \in f(\mathbb{N})} \sum_{k}\left|\sum_{n \in N}\left(a_{n k}-a_{(n-1) k}\right)\right|<\infty,
$$

where $f(\mathbb{N})$ denotes the collection of all finite subsets of $\mathbb{N}$.

Proof. First, we observe that $b v=\ell_{1 \Delta}$, where $\Delta$ is the difference operator $B^{-1}$ of Example 14. The inverse of $\Delta$ is $B$. By Corollary 11, $A \in\left(\ell_{\infty}, \ell_{1 \Delta}\right)$ if and only if $\Delta \circ A \in\left(\ell_{\infty}, \ell_{1}\right)$. Since $b v \subset c$, we have $A \in\left(\ell_{\infty}, c\right)$ and so by the Schur Theorem (see, e.g., Theorem 2.11 of [15]) rows of $A$ are in $\ell_{1}$. By Theorem 3 part (ii), $\Delta$ and $A$ are associative over $\ell_{\infty}$. So, $\Delta \circ A=\Delta A$ over $\ell_{\infty}$. We have

$$
[\Delta A]_{n k}=a_{n k}-a_{(n-1) k}
$$

where $a_{0 k}=0$ for all $k \in \mathbb{N}$. Now, using Theorem 2.14 of [15], we have $A \in\left(\ell_{\infty}, b v\right)$ if and only if

$$
\sup _{N \in f(\mathbb{N})} \sum_{k}\left|\sum_{n \in N}\left(a_{n k}-a_{(n-1) k}\right)\right|<\infty .
$$

Using Theorem 12, we have the following.

Corollary 16. If $A=\left(a_{n k}\right) \in\left(\ell_{\infty}, b v\right)$, then $A=\left(a_{n k}\right) \in$ $\mathscr{B}\left(\ell_{\infty}, b v\right)$ and

$$
\|A\|_{\left(\ell_{\infty}, b v\right)}=\|\Delta A\|_{\left(\ell_{\infty}, \ell_{1}\right)} .
$$

Example 17. Let $T=\left(t_{n k}\right)$ be a row finite matrix and $C$ the Cesàro operator which has a matrix representation

$$
C=\left[\begin{array}{ccccc}
1 & 0 & 0 & 0 & \cdots \\
1 / 2 & 1 / 2 & 0 & 0 & \cdots \\
1 / 3 & 1 / 3 & 1 / 3 & 0 & \cdots \\
1 / 4 & 1 / 4 & 1 / 4 & 1 / 4 & \ldots \\
\vdots & \vdots & \vdots & \vdots & \ddots
\end{array}\right]
$$

Then, $c_{C}$ is the well-known space of Cesàro summable sequences. This is a sequence space which includes the space of convergent sequences $c$. $C$ is invertible and the inverse operator has the matrix representation

$$
C^{-1}=\left[\begin{array}{ccccc}
1 & 0 & 0 & 0 & \cdots \\
-1 & 2 & 0 & 0 & \cdots \\
0 & -2 & 3 & 0 & \cdots \\
0 & 0 & -3 & 4 & \cdots \\
\vdots & \vdots & \vdots & \vdots & \ddots
\end{array}\right] .
$$

Since $T, C$, and $C^{-1}$ are all row finite, the operator $C \circ T \circ C^{-1}$ is represented by the matrix $C T C^{-1}$. Now, using Corollary 11 , we have that $T$ is in $\left(\left(\ell_{\infty}\right)_{C},\left(\ell_{\infty}\right)_{C}\right)$ if and only if $C T C^{-1}$ is in $\left(\ell_{\infty}, \ell_{\infty}\right)$, where $\left(\ell_{\infty}\right)_{C}$ denotes the space, introduced by $\mathrm{Ng}$ and Lee [3], of all sequences whose $C$-transforms are in the space $\ell_{\infty}$.

Let $s_{n k}$ be defined as in the previous example. Then, after some calculations, we have

$$
\left[C T C^{-1}\right]_{n k}=\frac{k}{n}\left(s_{n k}-s_{n(k+1)}\right) .
$$

Then, $C T C^{-1}$ is in $\left(\ell_{\infty}, \ell_{\infty}\right)$ if and only if

$$
\sup _{n} \sum_{k} \frac{k}{n}\left|s_{n k}-s_{n(k+1)}\right|<\infty
$$

by Theorem 2.6 of [15]. So, we have $T \in\left(\left(\ell_{\infty}\right)_{C},\left(\ell_{\infty}\right)_{C}\right)$ if and only if (27) holds. Similarly, we have $T \in\left(c_{C},\left(\ell_{\infty}\right)_{C}\right)$ or $T \in\left(\left(c_{0}\right)_{C},\left(\ell_{\infty}\right)_{C}\right)$ if and only if (27) holds, where $\left(c_{0}\right)_{C}$ and $c_{C}$ denote the spaces $\widetilde{c_{0}}$ and $\widetilde{c}$, studied by Şengönül and Başar [4], of all sequences whose $C$-transforms are in the spaces $c_{0}$ and $c$, respectively.

Theorem 18. Let $C$ be the Cesàro matrix. Then, $A=\left(a_{n k}\right) \in$ $\left(c, c_{C}\right)$ if and only if

$$
\begin{gathered}
\sup _{n}\left[\frac{1}{n} \sum_{k}\left|s_{n k}\right|\right]<\infty, \\
\lim _{n} \frac{s_{n k}}{n} \quad \text { exists for each } k, \\
\lim _{n} \frac{1}{n} \sum_{k} s_{n k} \quad \text { exists, }
\end{gathered}
$$

where $s_{n k}=a_{1 k}+a_{2 k}+\cdots+a_{n k}$.

Proof. By Corollary 11, $A \in\left(c, c_{C}\right)$ if and only if $C \circ A \in(c, c)$. Since $A \in\left(c, c_{C}\right) \subset(c, \omega)$, we have that rows of $A$ are in $\ell_{1}$. By Theorem 3 part (ii), $C$ and $A$ are associative over $c$. So, $C \circ A=$ $C A$ over $c$. We have

$$
[C A]_{n k}=\frac{s_{n k}}{n} .
$$

Now, using Kojima-Schur Theorem, we have $A \in\left(c, c_{C}\right)$ if and only if conditions (28) hold.

Lemma 19. If $\sum_{k=1}^{\infty} a_{k} c_{k}$ is convergent for all Cesàro summable sequences $\left(c_{k}\right)$, then $\lim k a_{k}=0$.

Proof. Suppose that $\lim k a_{k} \neq 0$. Then, there exist $\epsilon>0$ and a subsequence $\left(a_{k_{n}}\right)$ of $\left(a_{k}\right)$ such that $k_{n}\left|a_{k_{n}}\right|>\epsilon$. Without loss of generality, we can choose this subsequence $\left(a_{k_{n}}\right)$ such that $k_{n} \geq n^{2} k_{n-1}$. Let

$$
c_{k}= \begin{cases}\frac{k_{n}\left|a_{k_{n}}\right|}{n a_{k_{n}}} & \text { if } k=k_{n} \\ 0 & \text { otherwise. }\end{cases}
$$


Then, the sequence $\left(c_{k}\right)$ is Cesàro summable because for $k_{n} \leq$ $m<k_{n+1}$ we have

$$
\begin{aligned}
\mid \frac{c_{1}+}{c_{2}+\cdots+c_{m}} & \\
& \leq \frac{\left|c_{1}\right|+\left|c_{2}\right|+\cdots+\left|c_{m}\right|}{m} \\
& =\frac{\left|c_{k_{1}}\right|+\left|c_{k_{2}}\right|+\cdots+\left|c_{k_{n}}\right|}{m} \\
& =\frac{k_{1} / 1+k_{1} / 2+\cdots+k_{n} / n}{m} \\
& \leq \frac{k_{1} / 1+k_{1} / 2+\cdots+k_{n-1} /(n-1)+k_{n} / n}{k_{n}} \\
& =\frac{k_{1}}{k_{n}}+\frac{k_{2}}{2 k_{n}}+\cdots+\frac{k_{n-1}}{(n-1) k_{n}}+\frac{1}{n} \\
& \leq \frac{1}{n^{2}}+\frac{1}{2 n^{2}}+\cdots+\frac{1}{(n-1) n^{2}}+\frac{1}{n} \\
& \leq(n-1) \frac{1}{n^{2}}+\frac{1}{n} \leq \frac{2}{n} .
\end{aligned}
$$

On the other hand,

$$
\sum_{k=1}^{\infty} a_{k} c_{k}=\sum_{n=1}^{\infty} a_{k_{n}} c_{k_{n}}=\sum_{n=1}^{\infty} \frac{k_{n}\left|a_{k_{n}}\right|}{n}>\epsilon \sum_{n=1}^{\infty} \frac{1}{n}
$$

and so we get to the contradiction $\sum_{k=1}^{\infty} a_{k} c_{k}=\infty$ for the Cesàro summable sequence $\left(c_{k}\right)$. So, our assumption $\lim k a_{k} \neq 0$ is not true.

Theorem 20. Let $C$ be the Cesàro matrix. Then, $A=\left(a_{n k}\right) \in$ $\left(c_{C}, c_{C}\right)$ if and only if

$$
\begin{gathered}
\lim _{k} k a_{n k}=0 \quad \text { for each } n, \\
\sup _{n} \sum_{k} \frac{k}{n}\left|s_{n k}-s_{n(k+1)}\right|<\infty, \\
\lim _{n} \frac{s_{n k}-s_{n(k+1)}}{n} \quad \text { exists for each } k, \\
\lim _{n} \frac{1}{n} \sum_{k} k\left(s_{n k}-s_{n(k+1)}\right) \quad \text { exists, }
\end{gathered}
$$

where $s_{n k}=a_{1 k}+a_{2 k}+\cdots+a_{n k}$.

Proof. Suppose that $A \in\left(c_{C}, c_{C}\right)$. Then, by Lemma 19, we have (33). By Corollary 11, $A \in\left(c_{C}, c_{C}\right)$ if and only if $C \circ A \circ C^{-1} \in$ $(c, c)$. First let us show that $A$ and $C^{-1}$ are associative over $c$. Let $x=\left(x_{k}\right) \in c$. We have that

$$
\left(A\left(C^{-1} x\right)\right)_{n}=\sum_{k=1}^{\infty} a_{n k}\left(k x_{k}-(k-1) x_{k-1}\right)
$$

is convergent in $\mathbb{C}$. Let $S_{m}$ be the $m$ th partial sum of this series. Then,

$$
\begin{aligned}
S_{m} & =\sum_{k=1}^{m-1} k\left(a_{n k}-a_{n(k+1)}\right) x_{k}+m a_{n m} x_{m} ; \\
\lim _{m} m a_{n m}=0 \text {, so } & \\
\left(A\left(C^{-1} x\right)\right)_{n} & =\lim _{m} S_{m}=\sum_{k=1}^{\infty} k\left(a_{n k}-a_{n(k+1)}\right) x_{k} \\
& =\left(\left(A C^{-1}\right) x\right)_{n} ;
\end{aligned}
$$

$A C^{-1} \in(c, \omega)$ and $C$ is row finite. Then, by Theorem 3 part (ii), $C$ and $A C^{-1}$ are associative over $c$. So, $C \circ A \circ C^{-1}=C A C^{-1}$ over $c$. We have

$$
\left[C A C^{-1}\right]_{n k}=\frac{k}{n}\left(s_{n k}-s_{n(k+1)}\right) .
$$

Now, using Kojima-Schur Theorem, $A \in\left(c_{C}, c_{C}\right)$ implies conditions (34)-(36).

For the reverse implication, suppose that conditions (33)(36) hold. Then, by the Kojima-Schur Theorem, conditions (34)-(36) imply $C A C^{-1} \in(c, c)$. By (33), $A \circ C^{-1}=A C^{-1}$ over $c$. By Part (ii) of Theorem 3, $C$ and $A C^{-1}$ are associative over $c$, and so we have $C \circ A \circ C^{-1}=C A C^{-1}$ over $c$. Hence, $C \circ A \circ C^{-1} \in(c, c)$, and now by Corollary $11, A \in\left(c_{C}, c_{C}\right)$.

Corollary 21. If $A=\left(a_{n k}\right) \in\left(c_{C}, c_{C}\right)$, then $A=\left(a_{n k}\right) \in$ $\mathscr{B}\left(c_{C}, c_{C}\right)$ and

$$
\|A\|_{\left(c_{C}, c_{C}\right)}=\left\|C A C^{-1}\right\|_{(c, c)}=\sup _{n} \sum_{k} \frac{k}{n}\left|s_{n k}-s_{n(k+1)}\right| .
$$

Finally, let one give an application on a matrix operator which is not a triangle.

Theorem 22. Let

$$
\Gamma=\left[\begin{array}{ccccc}
\gamma_{1} & \gamma_{2} & \gamma_{3} & \gamma_{4} & \cdots \\
0 & \gamma_{2} & \gamma_{3} & \gamma_{4} & \cdots \\
0 & 0 & \gamma_{3} & \gamma_{4} & \cdots \\
0 & 0 & 0 & \gamma_{4} & \cdots \\
\vdots & \vdots & \vdots & \vdots & \ddots
\end{array}\right],
$$

where $\gamma_{k} \neq 0$ for all $k \in \mathbb{N}$. Then, a matrix $A \in\left(c_{0 \Gamma}, c_{0}\right)$ if and only if the following three conditions hold:

$$
\begin{gathered}
\left(\frac{a_{n k}}{\gamma_{k}}\right)_{k=1}^{\infty} \text { is bounded for each } n, \\
\sup _{n} \sum_{k=1}^{\infty}\left|\frac{a_{n k}}{\gamma_{k}}-\frac{a_{n(k-1)}}{\gamma_{k-1}}\right|<\infty, \\
\lim _{n}\left(\frac{a_{n k}}{\gamma_{k}}-\frac{a_{n(k-1)}}{\gamma_{k-1}}\right)=0 \quad \text { for each } k,
\end{gathered}
$$

where $a_{n 0} / \gamma_{0}=0$. 
Proof. Suppose that $A \in\left(c_{0 \Gamma}, c_{0}\right)$. The operator $\Gamma: \omega_{\Gamma} \rightarrow \omega$ is one to one, because for $\Gamma x=0$ with $x=\left(x_{k}\right) \in \omega$ we have the system of equations

$$
\begin{gathered}
0=\sum_{j=k}^{\infty} \gamma_{j} x_{j} \quad \text { for } k=1,2,3, \ldots, \\
0=\gamma_{k} x_{k}+\sum_{j=k+1}^{\infty} \gamma_{j} x_{j}=\gamma_{k} x_{k}+0=\gamma_{k} x_{k} \quad \text { for } k=1,2,3, \ldots
\end{gathered}
$$

Now, let us show that $\Gamma: c_{0 \Gamma} \rightarrow c_{0}$ is onto. Let $y=\left(y_{k}\right) \epsilon$ $c_{0}$. Then $\Gamma x=y$ for $x=\left(x_{k}\right)$ with $x_{k}=\gamma_{k}^{-1}\left(y_{k}-y_{k+1}\right)$ since

$$
\begin{aligned}
(\Gamma x)_{n} & =\gamma_{n} x_{n}+\gamma_{n+1} x_{n+1}+\cdots \\
& =\left(y_{n}-y_{n+1}\right)+\left(y_{n+1}-y_{n+2}\right)+\cdots=y_{n} .
\end{aligned}
$$

Hence, $\Gamma: c_{0 \Gamma} \rightarrow c_{0}$ is onto. So, $\Gamma: c_{0 \Gamma} \rightarrow c_{0}$ is bijective. The inverse operator is then

$$
\Gamma^{-1}=\left[\begin{array}{ccccc}
\gamma_{1}^{-1} & -\gamma_{1}^{-1} & 0 & 0 & \cdots \\
0 & \gamma_{2}^{-1} & -\gamma_{2}^{-1} & 0 & \ldots \\
0 & 0 & \gamma_{3}^{-1} & -\gamma_{3}^{-1} & \ldots \\
0 & 0 & 0 & \gamma_{4}^{-1} & \ldots \\
\vdots & \vdots & \vdots & \vdots & \ddots
\end{array}\right] .
$$

Now, by Theorem 10, $A \in\left(c_{0 \Gamma}, c_{0}\right)$ if and only if $A \circ \Gamma^{-1} \in$ $\left(c_{0}, c_{0}\right)$.

For $x=\left(x_{k}\right) \in c_{0}$ we have $\Gamma^{-1} x=\left(\gamma_{1}^{-1}\left[x_{1}-x_{2}\right], \gamma_{2}^{-1}\left[x_{2}-\right.\right.$ $\left.\left.x_{3}\right], \ldots\right)$, and then for $A=\left(a_{n k}\right)$ we have $\left(A\left(\Gamma^{-1} x\right)\right)_{n}=$ $\sum_{k} a_{n k} \gamma_{k}^{-1}\left(x_{k}-x_{k+1}\right)$. Let $S_{m}$ be the $m$ th partial sum of this series. Then,

$$
S_{m}=\sum_{k=1}^{m}\left(a_{n k} \gamma_{k}^{-1}-a_{n(k-1)} \gamma_{k-1}^{-1}\right) x_{k}-a_{n m} \gamma_{m}^{-1} x_{m+1}
$$

where $a_{n 0}=0$.

By a similar proof to the proof of Lemma 19, we can see that the sequence $\left(a_{n m} \gamma_{m}^{-1}\right)_{m=1}^{\infty}$ is bounded for each $n$, when $A \in\left(c_{0 \Gamma}, \omega\right)$. Then,

$$
\begin{aligned}
\left(A\left(\Gamma^{-1} x\right)\right)_{n} & =\lim _{m} S_{m} \\
& =\sum_{k=1}^{\infty}\left(a_{n k} \gamma_{k}^{-1}-a_{n(k-1)} \gamma_{k-1}^{-1}\right) x_{k}=\left(\left(A \Gamma^{-1}\right) x\right)_{n}
\end{aligned}
$$

The conditions for $A \Gamma^{-1}$ to be in $\left(c_{0}, c_{0}\right)$, by Example $5 \mathrm{~A}$ page 129 of [12], are

$$
\begin{aligned}
& \sup _{n} \sum_{k=1}^{\infty}\left|a_{n k} \gamma_{k}^{-1}-a_{n(k-1)} \gamma_{k-1}^{-1}\right|<\infty, \\
& \lim _{n}\left(a_{n k} \gamma_{k}^{-1}-a_{n(k-1)} \gamma_{k-1}^{-1}\right)=0 \text { for each } k .
\end{aligned}
$$

Hence, we have all conditions (43). We leave the reverse implication part to the reader.

\section{Acknowledgment}

The author thanks the referees for their valuable comments and suggestions.

\section{References}

[1] H. Kizmaz, "On certain sequence spaces," Canadian Mathematical Bulletin, vol. 24, no. 2, pp. 169-176, 1981.

[2] S. M. Sirajudeen, "Matrix transformations of $b v$ into $l(q), l_{\infty}(q)$, $c_{0}(q)$ and $c(q)$," Indian Journal of Pure and Applied Mathematics, vol. 23, no. 1, pp. 55-61, 1992.

[3] P. N. Ng and P. Y. Lee, "Cesàro sequence spaces of non-absolute type," Commentationes Mathematicae (Prace Matematyczne), vol. 20, no. 2, pp. 429-433, 1977/78.

[4] M. Şengönül and F. Başar, "Some new Cesàro sequence spaces of non-absolute type which include the spaces $c_{0}$ and $c$," Soochow Journal of Mathematics, vol. 31, no. 1, pp. 107-119, 2005.

[5] B. Altay and F. Başar, "Some Euler sequence spaces of nonabsolute type," Ukrainian Mathematical Journal, vol. 57, no. 1, pp. 3-17, 2005.

[6] B. Altay, F. Başar, and M. Mursaleen, "On the Euler sequence spaces which include the spaces $l_{p}$ and $l_{\infty}$. I," Information Sciences, vol. 176, no. 10, pp. 1450-1462, 2006.

[7] M. Mursaleen, F. Başar, and B. Altay, "On the Euler sequence spaces which include the spaces $l_{p}$ and $l_{\infty}$. II," Nonlinear Analysis: Theory, Methods \& Applications, vol. 65, no. 3, pp. 707717, 2006.

[8] C. S. Wang, "On Nörlund sequence spaces," Tamkang Journal of Mathematics, vol. 9, no. 2, pp. 269-274, 1978.

[9] E. Malkowsky and V. Rakočević, "On matrix domains of triangles," Applied Mathematics and Computation, vol. 189, no. 2, pp. 1146-1163, 2007.

[10] I. Djolović and E. Malkowsky, "A note on compact operators on matrix domains," Journal of Mathematical Analysis and Applications, vol. 340, no. 1, pp. 291-303, 2008.

[11] J. Boos, C. Stuart, and C. Swartz, "Gliding hump properties of matrix domains," Analysis Mathematica, vol. 30, no. 4, pp. 243257, 2004.

[12] A. Wilansky, Summability through Functional Analysis, vol. 85 of North-Holland Mathematics Studies, North-Holland, Amsterdam, The Netherlands, 1984.

[13] M. Altun, "Matrix mappings on multiplier sequence spaces," Thai Journal of Mathematics, vol. 11, no. 1, pp. 59-66, 2013.

[14] B. Altay and F. Başar, "On the fine spectrum of the generalized difference operator $B(r, s)$ over the sequence spaces $c_{0}$ and c," International Journal of Mathematics and Mathematical Sciences, vol. 2005, no. 18, pp. 3005-3013, 2005.

[15] I. J. Maddox, Infinite Matrices of Operators, vol. 786 of Lecture Notes in Mathematics, Springer, Berlin, Germany, 1980. 


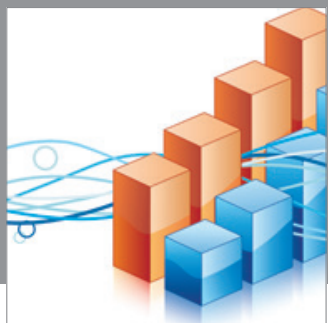

Advances in

Operations Research

mansans

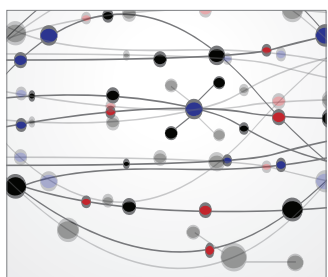

The Scientific World Journal
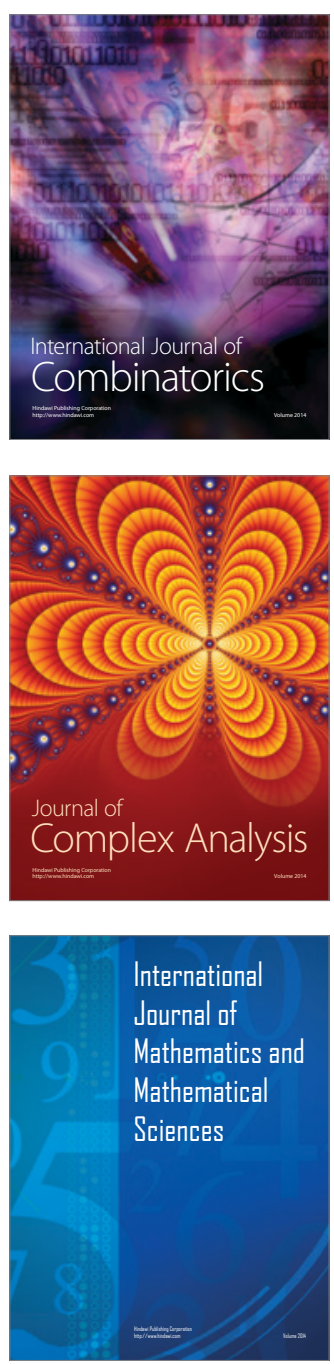
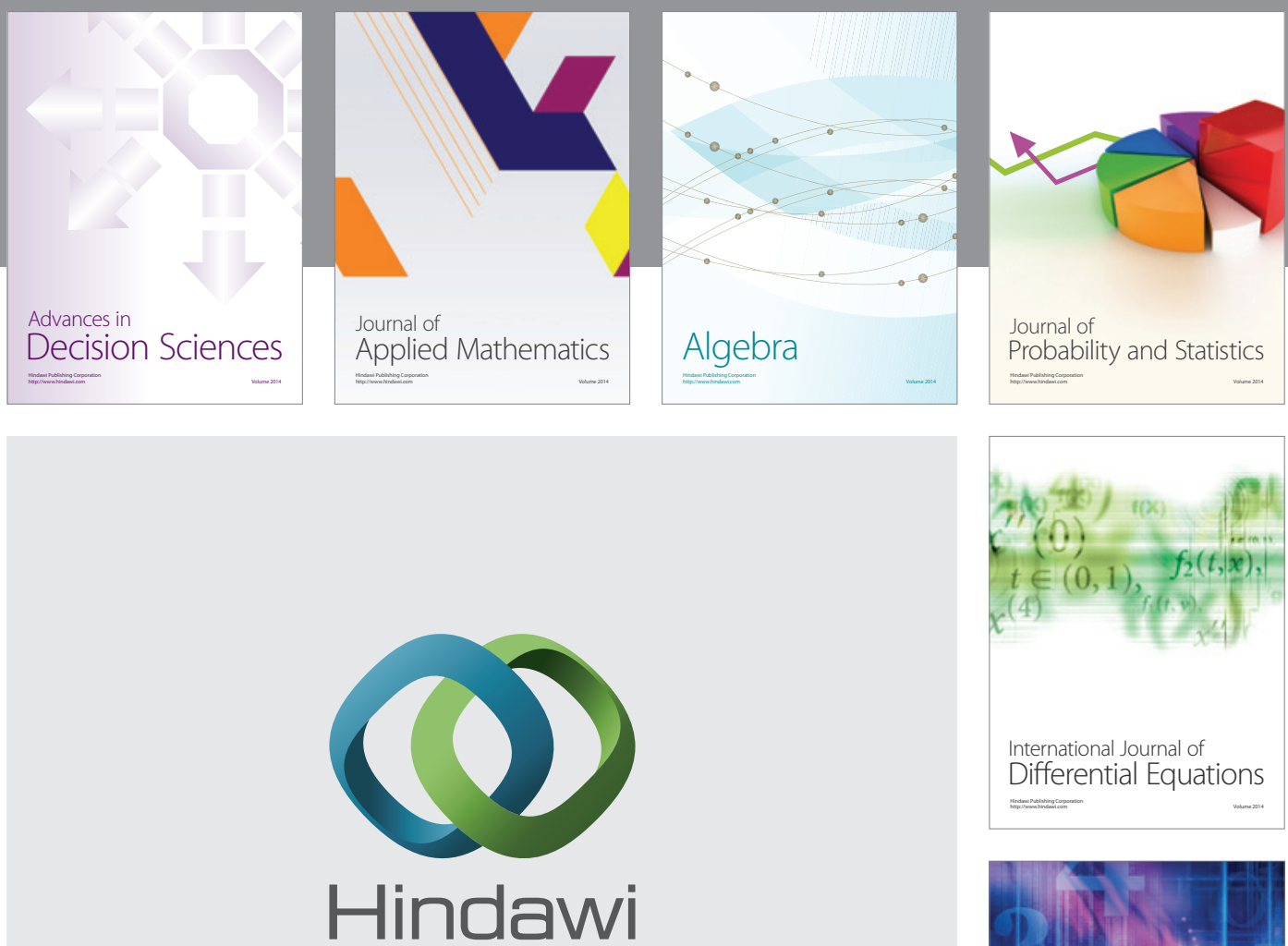

Submit your manuscripts at http://www.hindawi.com
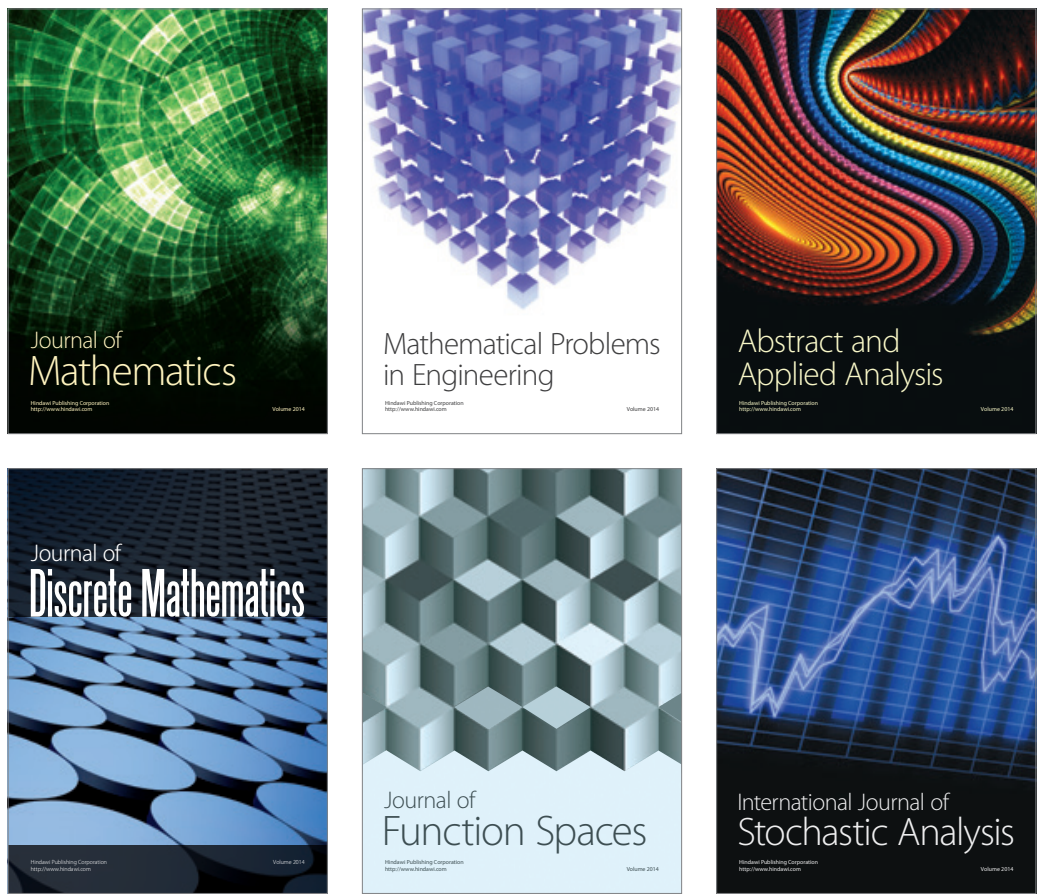

Journal of

Function Spaces

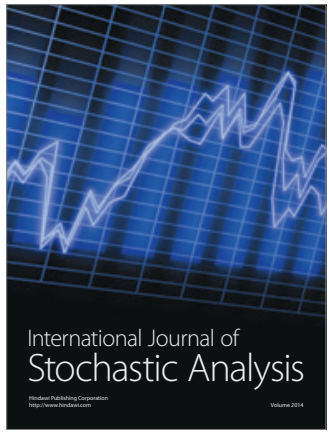

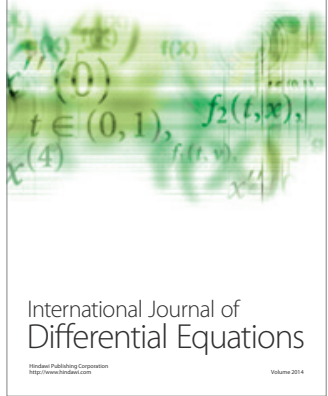
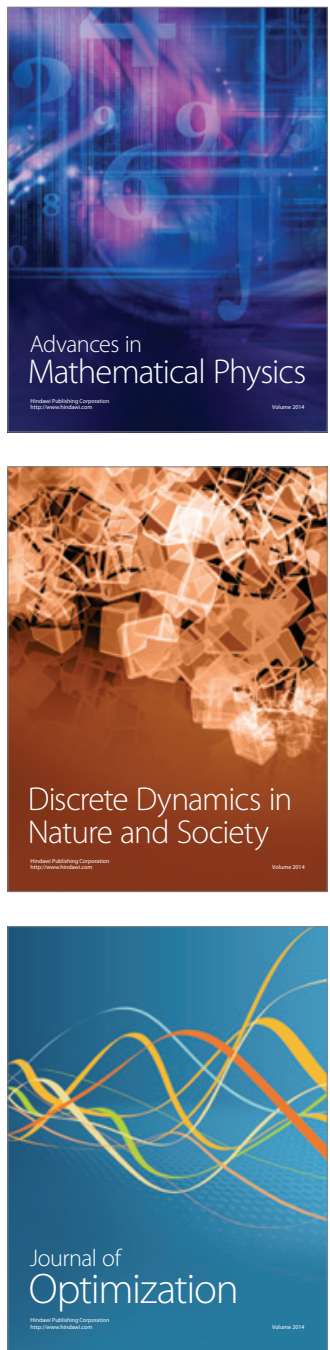